\title{
Establishment of Public Cord Blood Banks - The Need of the Hour in India
}

\author{
Authors \\ Suriya Narayanan $S^{1}$, Jaianand Kannaiyan ${ }^{2}$, Palaniyandi $\mathbf{M}^{3}$, Rajangam $\mathbf{B}^{4}$, Bhavna $\mathrm{A}^{5}$, \\ Anubhav Pandey ${ }^{6} *$ \\ ${ }^{1}$ Head, Laboratory Operations, ${ }^{2}$ Head, Clinical Research, ${ }^{3}$ Asst. Manager, Tissue Culture, \\ ${ }^{4}$ Manager, Tissue Culture, ${ }^{5}$ Deputy General Manager, Medical Affairs, ${ }^{6}$ Medical Director \\ CelluGen Biotech Private Limited, Gurgaon-122016, Haryana, India \\ Corresponding Author \\ Dr. Anubhav Pandey \\ Medical Director, CelluGen Biotech Private Limited, CelluGen House, 62, Udyog Vihar Phase-1, \\ Gurgaon- 122016, Haryana, India \\ Email: dranubhav.pandey@cellugen.in, Contact: +91-7838515178
}

\begin{abstract}
Since, the first successful transplantation of umbilical cord blood in 1988, marking 25th anniversary of the first umbilical cord blood transplantation performed in France, in a child with Fanconi Anemia, umbilical cord blood transplantation is considered as an alternative to hematopoietic stem cell source for patients with hematologic diseases. With over 600,000 umbilical cord blood units stored for transplantation worldwide and over 30,000 umbilical cord blood transplantation performed, the patient pool in India treated with umbilical cord blood transplant using related or unrelated is merely only 32. In a country, where private cord blood banking has a significant attention and considering the large number of patients born with blood related disorders, these transplantations are comparatively few. In this paper, we review the need of setting up of a public cord blood bank across the country, lack of sufficient umbilical cord blood repository, therapeutic challenges associated with umbilical cord blood with an increase in hematological diseases incidence in India. Keywords- Private cord blood banks; Public cord blood banks; Stem cell transplantation; Umbilical cord blood banking.
\end{abstract}

\section{Introduction}

Hematopoietic cell Transplantation (HCT) is one of the widely accepted modes of treatment choice in children and adults suffering from various malignant and non malignant disorders either acquired or inherited. HCT is also used for the patients with solid tumors undergoing high dose chemotherapy helping the limit of drug administration and its associated toxicities in cases like soft tissue sarcomas, neuroblastoma and germ cell tumors. ${ }^{1}$
The enormous progress made in the field of alternative donor bone marrow transplantation (BMT) has not yet touched the Indian shores. In India autologous and related bone marrow transplantation is performed since 1981, however only 300 procedures are done yearly by centers in 20 major cities across the country. It is reported that $70 \%$ of the patients in India cannot find matched donors from their family, siblings and have to look for unrelated matched donors. ${ }^{2}$ The scenario for Indians diagnosed with hematological conditions 
living in India and settled overseas, suffer due to lack of sufficient unrelated marrow registries and public cord blood repositories. Only less than $10 \%$ chances are there for an Indian to find a matched donor from international registries and even if found the cost of the procuring the donor units from the international registries are very high. 3 In India, with over 1.3 crores of diverse ethnic and genetically varied population, getting a matched bone marrow donor is relatively a herculean task.

The umbilical cord blood (UCB) cells are being biologically different, it requires a $4 / 6$ match as opposed to 6/6 match required for $\mathrm{BM}$ transplantation. Moreover, cryo-preserved UCB is readily available, can be easily shipped and thawed for use when needed alleviating the problem of donor attrition. These exciting cord blood stem cells has led to clinical trials using $\mathrm{CB}$ to treat various diseases including cerebral palsy, brain injury, and juvenile diabetes. ${ }^{2-3}$

This article focuses on the establishment of Public Cord Blood Bank. We also discuss the Indian scenario of umbilical cord blood transplantation and the need for public cord blood bank repositories, to create awareness to both public and government regulators to set up public cord blood banks to meet the demand of cord blood units.

\section{Umbilical Cord Blood Transplantation}

The first successful umbilical cord blood transplantation (UCBT) was performed in 5 year old patient with Fanconi's Anemia (FA). Gluckman et al described hand delivered cord blood stem cells shipped in dry shipper were thawed; cell viability and Hematopoietic Progenitor Cells (HPC's) count were done before transplantation. The UCBT Engraftment was successful and no Graft Versus Host Disease (GVHD) was noticed. The patient who underwent the first successful UCBT in 1988 is reported healthy even after 25 years of transplantation with complete hematological and immunological donor reconstitution. ${ }^{4-5}$

Since then, Cord Blood stem cells have been successfully used in transplant medicine for more than 20 years. To date, UCB has been used to treat nearly 80 serious diseases, including leukemia, other cancers, and blood disorders. CB is being researched now for use in regenerative medicine where stem cells may help induce healing or regenerate cells to repair tissues.

\section{Hematological Conditions: An Indian Context}

To date, nearly 80 different diseases are being treated using cord blood stem cells, namely sickle cell anaemia, thalassemia and other haematopoietic disorders. In these, sickle cell anaemia is considered major health issue affecting 5,200 live births each year. Mostly affected are tribal populations ranging upto 5-34\% causing severe socio-economic disadvantages. Thalassemia is also one of the main disorders and estimated that about $10 \%$ of the world thalassemic people live in Indian sub-continent. Furthermore, among the child hood cancers, leukemia is the prevalent of all, affecting $25-45 \%$ of children in India. Sixty to $85 \%$ of all leukemias reported are Acute Lymphoblastic Leukemia (ALL). Compared to the developed world, the biology of ALL appears different in India, with a higher proportion of T-Cell ALL, which contributes to poorer prognosis of leukemia. ${ }^{6}$

In India, Leukemia is the most common childhood cancer with $25 \%$ to $40 \%$ of children suffering with 60-85\% being Acute Lymphoid Leukemia (ALL). National Cancer Registry Programme (NCRP) in 2009 conducted a study in five urban registries and one rural registry across India, the study indicates the changes in terms of incidence rates of lymphoid and hematological cancers across six registries with 104,239 cases of leukemia reported in 2010 and estimated to increase to 132,574 in $2020 .^{6}$

The incidence of Thalassemia in India is rapidly on the rise with at least 10,000 children affected with Thalassemia major every year. Twenty million carriers with thalassemia are also reported. Aplastic anemia, a clinical condition where the body stops producing enough red blood cells is also on a rise in India particularly affecting 6 to 8 million population. ${ }^{7}$ 


\section{Private UCB Bank: An Unidentified Possible Future}

While there has been ground breaking research on UCBT for battling diseases such as leukaemia, sickle cell anaemia and thalassemia, non-availability of matched donor is a major obstacle in India. ${ }^{8}$ Private cord blood banking in India is gaining significance as people are aware that storing their umbilical cord blood can save their baby's future ailments, however they fail to understand and realize the stored baby's umbilical cord blood will provide no answer if their baby is diagnosed with disease of genetic origin. Private facilities provide an opportunity to donors to store their cord blood, hoping in the future, a member of their family may use it for treating degenerative diseases. However, no clear evidence available that their future use will be feasible or efficacious or not.

Even though private cord blood banks store child's cord blood for twenty years, the volume of cord blood stored for transplantation purpose may not guarantee the effective weight per kilogram requirement of stem cells for diseased child's need. In such a scenario public cord blood bank can combine one or more cord blood unit for transplantation which has been recommended for hematopoietic blood transplantations. In most blood related disorders, transplant specialists recommend Umbilical Cord Blood Transplant (UCBT) to be of another person (allogenic) and not their own (autologous). Parents should be educated by the fact that child's own cord blood can rarely be used for transplant. This is because over $90 \%$ of the blood related disorders in child are from the time of birth and the cord blood stem cells of child will carry the same mutated gene and if transplanted to child, will confer same gene to child.8 In scenarios like this, unrelated matched cord units from the public cord blood repositories can be used to save the children diagnosed with pediatric cancers. The therapeutic utility of UCBT for personal use is being overstated by private banks to make impact on their commercial end.

European countries such as Italy, France and Belgium have banned unlawful storage of cord blood in private cord blood banks. ${ }^{9-10}$ Even,
European group on ethics in Science and New Technologies recommended European Commission that the authenticities of private cord blood banks storing autologous cord blood should be questioned; they are marketing as service, which presently has no real usage in regards to therapeutic applications. They recommend that support for public cord blood banks for allogeneic transplantations should be increased and long-term functioning should be assured. $^{10}$

The Society of Obstetricians and Gynecologists of Canada Clinical Practice Guidelines and American Academy of Pediatrics clearly stated that, because of limited usage and lack of scientific support, collection and long-term storage of UCB for autologous donation is not recommended. ${ }^{11-12}$

\section{Lack of Public Cord Blood Repositories in India}

The cord blood units stored in private banks worldwide is almost approximately 6 times more than the public cord blood units, yet more than 30 times cord blood units are released for therapy from public cord bloods compared to private banks. Moreover, there are at least 142 public banks worldwide storing over 600,000 UCB units for transplantation. From these banks, over 30,000 successful transplants have been performed. Major public cord blood banks are set up in USA, Europe and Australia catering to ethnicities prevalent in those areas. ${ }^{13}$

Conversely in India, only 3 public banks, collectively storing only 7600 UCB units and treating 125 patients. The main reason behind low transplantation rate is due to genetic diversity and acute shortage of HLA matching. Even if matched, the cost of import would be at least USD 30,000 per unit. Moreover, no financial support given by the Government of India in this sector in comparison to North America where over USD 80 million has been contributed by the government.

In a country with 28 million births and nine million blood donations annually, the number of donated umbilical cord blood units in public cord blood banks is very minimal and India's global share in cord blood based surgical treatment is a meager $0.25 \% .^{7}$ 
The reason for very less stored public umbilical cord units is primarily due to (i) lack of awareness in the Indian population (ii) lack of funding from the government bodies to fund the public cord blood banks to increase their pool size so matching of unrelated donors UCB units can be found when needed. (Only Government of Tamilnadu has given a grant to a public cord blood bank operating in Chennai, India) (iii) lack of knowledge of physicians about rationale for cord blood banking and difference between private and public cord blood banks, (iv) The lack of stringent guidelines emphasizing the importance of minimum volume of cord blood to be collected from the donor to address $20-30 \times 106$ nucleated cells $/ \mathrm{kg}$ or more than $1.25 \mathrm{x}$ 106 CD34+ cells (v) lack of encouragement to store the cord blood in public banks (vi) lack of information provided by the banks regarding the limitations of autologous and allogenic cord blood transplantation.

\section{The Need of the Hour}

According to a 2009 study, the modern Indian populations are composed of two distinct genetically divergent (Indo-Aryan and Dravidian) and heterogeneous populations. This genetic diversity factor limits the probability of finding HLA match for patients of Indian origin in foreign registries. India needs to develop its own pool of UCB units to find HLA matched UCBs for its patients. India has a high birth rate of 26 million births per year with a genetically diverse population. This positions our country to be probably the largest pool of genetically diverse UCB units in the world.

Currently, only two public cord blood banks are operational in India, to store umbilical cord blood units from volunteers. The increase in numbers also emphasize the need of more public cord blood banks in India to meet the high demand and to solve the umbilical cord blood requests across the country. However there are close to 15 umbilical cord blood stem cell banks storing a large portion of private umbilical cords, the utilization of which is highly debatable. The need of the hour is to increase the availability of UCB units through public bank sector for donor transplant in India thereby increasing the number of cord blood unit repository. ${ }^{14-15}$

\section{Conclusion}

There is a steady increase in cord blood transplants globally due to non-availability of compatible bone marrow donor. With high success rate, cord blood transplantation is the only approved form of stem cell therapy and covered by medical insurance. The awareness on umbilical cord blood storage and its importance should be imparted to all pregnant mothers, gynecologist and transplant physicians. Since cord blood units collected from the umbilical cord blood with genetic disorder of leukemia, thalassemia and sickle cell anemia will not be useful to the child for autologous UCBT, in such cases the access for the units from public cord blood bank repositories are essential. Thus, public cord blood bank with sizeable number of repositories and government assistance in regulating and funding would suffice the transplantation need.

\section{Acknowledgement}

Authors would like to express sincere gratitude to Dr. Firdosh Mahuvawala and Mr. Lalit Jaiswal, Directors for the encouragement and authors are pleased to Mr. Anand Bhushan, Chief Operating Officer in guiding throughout the study.

\section{References}

1. Appelbaum FR. Pursuing the goal of a donor for everyone in need. N Engl J Med. 2012; 367(16):1555-1556.

2. Chandra V, Arunava R, Devi D, Arun Kumar M, Preeti K. Optimization of the Inventory size of the Public cord blood program- The Indian context. The Journal of the Association of Physicians of India. 2010; 58: 608-611

3. Pitchappan RM, Kavitha, Jayalakshmi M. HLA genomic diversity of India and its implications in HIV pandemic. Intl J Hum Genet. 2008; 8: 143-153.

4. Broxmeyer HE, Douglas GW, Hangoc G, Copper S, Bard J et al. Human umbilical cord blood as a potential source of 
transplantable hematopoietic stem/progenitor cells. Proc Natl Acad Sci. 1988; 86(10): 3828-3832.

5. Eliane Gluckman, Broxmeyer Hal E, Arleen D, Auerbach, Henry S et al. Hematopoietic reconstitution in a patient with Fanconi's anemia by means of umbilical-cord blood from an HLA identified sibling. N Engl J Med. 1989; 32(17): 1174-1178.

6. Ramnath Takiar, Deenu Nadayil, Nandakumar. Projections of Number of Cancer Cases in India (2010-2020) by Cancer Groups. Asian Pacific Journal of Cancer Prevention. 2010; 11:1045-1049.

7. Beatty PG, Boucher KM, Milford EL. Probability of finding HLA-mismatched related or unrelated marrow or cord blood donors. Human Immunology 2000; 61:834-40.

8. Mckenna D, Sheth J. Umbilical cord blood: Current status \& promise for the future. Indian J Med Res 2011; 134:261-9.

9. Clea Caulcutt, Tom Cochez. Working grant International Offensive on Cord Blood Banking; Journalismfund.eu. File number: 2010/010.

10. Ethical aspects of umbilical cord blood banking: Opinion of the European Group of Ethics in Science and new Technologies to the European Commission. No 19; 2004.

11. McCullough J, McKenna D, Kadidlo D, Schierman T, Wagner J. Issues in the quality of umbilical cord blood stem cells for transplantation. Transfusion. 2005;45:832-41.

12. Armson BA. Maternal Fetal Medicine Committee, Society of Obstetricians and Gynecologists of Canada. Umbilical cord blood banking: implications for perinatal care providers. J Obstet Gynaecol Can. 2005;27:263-90.

13. Ballen KK, Veter F, Kurtzberg J. Umbilical cord blood donation: public or private? Bone Marrow Transplantation. 2015, 1-8.

14. Tomblyn MB, Arora M, Baker KS, et al. Myeloablative hematopoietic cell transplantation for acute lymphoblastic leukemia: analysis of graft sources and long- term outcome. Journal Clinical Oncology 2009; 27: 3634-41.

15. Jaing TH, Yang CP, Hung IJ, et al. Transplantation of unrelated donor umbilical cord blood utilizing double-unit grafts for five teenagers with transfusion dependant Thalassemia. Bone Marrow Transplant 2007; 40: 307-11. 